Original article

\title{
MAST CELL DISTRIBUTION AROUND THE NEEDLE TRACT FOLLOWING ACUPUNCTURE IN ZUSANLI (ST36) ACUPOINT IN RATS
}

\author{
N. D. DIMITROV ${ }^{1}$, D. Y. ATANASOVA ${ }^{1,3}$, N. S. TOMOV ${ }^{1}$, \\ Y. A. STAYKOVA-PIROVSKA ${ }^{2}$, I. G. IVANOVA ${ }^{1} \&$ D. P. SIVREV ${ }^{1}$ \\ ${ }^{1}$ Department of Anatomy; ${ }^{2}$ Department of General Medicine, \\ Faculty of Medicine, Trakia University, Stara Zagora, Bulgaria; \\ ${ }^{3}$ Institute of Neurobiology, Bulgarian Academy of Sciences, Sofia, Bulgaria
}

\section{Summary}

Dimitrov, N. D., D. Y. Atanasova, N. S. Tomov, Y. A. Staykova-Pirovska, I. G. Ivanova \& D. P. Sivrev, 2019. Mast cell distribution around the needle tract following acupuncture in Zusanli (ST 36$)$ acupoint in rats. Bulg. J. Vet. Med., 22, No 1, 91-98.

The aim of this study was to investigate mast cell (MCs) distribution in the vicinity of the needle tract formed after acupuncture in Zusanli ( $\mathrm{ST}_{36}$ ) acupoint in rats. MCs were detected by histochemistry, immunohistochemistry and transmission electron microscopy, and evaluated quantitatively. It was established that after acupuncture in $\mathrm{ST}_{36}$ acupoint the integrity of the epithelium, dermis, subcutaneous connective tissue, fascia, epimysium and striated muscles was disrupted and folded to the direction of the needle tract. In the thickened connective tissue MCs were observed close to the needle tract, without visible differences in their number along the tract, but most of them were with signs of degranulation, possibly due to acupuncture. It could be presumed that acupuncture in $\mathrm{ST}_{36}$ caused recruitment and activation of MCs followed by degranulation which most probably influenced the local microenvironment.

Key words: acupuncture, degranulation, mast cells, needle tract, Zusanli $\left(\mathrm{ST}_{36}\right)$

\section{INTRODUCTION}

Acupuncture is a commonly used method of the Traditional Chinese Medicine. Zusanli $\left(\mathrm{ST}_{36}\right)$ acupuncture point (acupoint) is one of the most important for treatment of both humans and animals. $\mathrm{ST}_{36}$ can be used in experimental acupuncture by applying the method of standard proportions of anatomical structures under the control of an apparatus measuring skin resistance (White et al., 2008; Dimitrov et al., 2009). Mast cells (MCs) are resident mainly in the connective tissue, particularly in vicinity of small blood vessels and nerves. Their usual localisation is in proximity to surfaces that interface the external environment. Biological 
functions of MCs include a role in innate immunity, mechanisms against parasitic infestations, immunomodulation of the immune system, and tissue repair (Metcalfe et al., 1997). The MCs are also important subject in experimental acupuncture. Many studies have been devoted to their role (Lin et al., 2007; Zhang et al., 2008).

Acupuncture point $\mathrm{ST}_{36}$ is one of frequently used points in experimental acupuncture in rats, often in combination with electroacupuncture (Deng et al., 1996; Ming et al., 2000; Li et al., 2003). Some studies combine experimental acupuncture with moxibustion (He \& Luo, 2007; Luo et al., 2007; He \& Chen, 2010). There is an evidence for a link between the effects of laser acupuncture and the function of MCs (Cheng et al., 2009). Our previous studies have shown that the normal anatomic structures in $\mathrm{ST}_{36}$ acupoint are the epidermis, dermis, subcutis, deep fascia, epimysium, striated muscle, containing blood vessels and nerves (Dimitrov, 2012a). The impact of the acupuncture needle in $\mathrm{ST}_{36}$ acupoint comprised morphological changes in the tissues and MCs. Following acupuncture in $\mathrm{ST}_{36}$ acupoint significant degranulation of $\mathrm{MCs}$ occurred in the acupuncture area (Dimitrov, 2012b).

The aim of this study was to study the distribution of MCs in the vicinity of the needle tract after acupuncture in $\mathrm{ST}_{36}$ acupoint in rats.

\section{MATERIALS AND METHODS}

\section{Animals}

The studies were carried out on ten adult male Wistar normotensive rats, weighing 220-350 g. The experimental design was approved by the Research Ethics Committee at the Medical Faculty of Trakia Uni- versity. All efforts were made to minimise the number of animals used and their suffering. The area around the acupoint $\mathrm{ST}_{36}$ was epilated, defined and marked using the method of standard proportions of anatomical structures under the control of the apparatus KWD-808 measuring skin resistance (Dimitrov, 2012b). A steel acupuncture needles with size $0.25 \times 13 \mathrm{~mm}$ were inserted in the $\mathrm{ST}_{36}$ of the ether narcotised rats just before perfusion of the experimental animals (from the left heart ventricle via aorta) with $0.05 \mathrm{M}$ phosphate buffered saline (PBS) followed by $4 \%$ of paraformaldehyde (PFA, Sigma Aldrich Chemie, Switzerland) in $0.1 \mathrm{M}$ phosphate buffer, $\mathrm{pH} 7.36$.

\section{Light microscopic histology for mast cells detection}

The material $(5 \times 5 \times 5 \mathrm{~mm})$ from $\mathrm{ST}_{36}$ acupoint was taken immediately after the death of the animals together with the acupuncture needle. Some of the samples were dehydrated in ascending ethanol series, cleared (twice) in xylene and embedded in paraffin. From them sections of $5 \mu \mathrm{m}$ thickness were prepared, rehydrated in descending ethanol series. Other sections with $30 \mu \mathrm{m}$ thickness were prepared on freezing microtome. Both sections were stained using toluidine blue (TB), Bismarck brown (BB) and Mallory's trichrome (MT) methods.

\section{Light microscopic immunohistochemistry for mast cells detection}

The paraffin sections with the same thickness were dewaxed twice in xylene and were rehydrated in descending ethanol series. Afterwards sections were washed in $0.1 \mathrm{M}$ PBS, $\mathrm{pH} 7.4$, incubated in $1.2 \%$ hydrogen peroxide in methanol for $30 \mathrm{~min}$, followed by antigen retrieval in 
$10 \mathrm{mM}$ citrate buffer ( $\mathrm{pH} 9.0$ ) for up to 10 min in pressure cooker.

Between the separate steps, the sections were rinsed with cold PBS/Triton X100. Subsequently, they were incubated with the primary antibody (Monoclonal Mast Cell Tryptase - clone 10D11, Leica Biosystems, Newcastle) diluted 1:100 in a humid chamber overnight at $4{ }^{\circ} \mathrm{C}$. Following three washings with $\mathrm{PBS}$, the slides were incubated with DAKO-REAL ${ }^{\mathrm{TM}}$ EnVision $^{\mathrm{TM}}$ detection system (DAKO) for 60 min, then visualised with diaminobenzidine and counterstained with Mayer's haematoxylin. PBS instead of the primary antibody was used as a negative control.

All slides were observed by a light microscope (Eclipse 80i, Nikon, Japan), analysed and photographed with a digital camera (Nikon DMX 1200).

\section{Transmission electron microscopy for mast cells detection}

The material $\left(1 \mathrm{~mm}^{3}\right)$ from the skin in the vicinity of the needle tract was fixed immediately in $2 \%$ glutaraldehyde and $2 \%$ paraformaldehyde in $0.1 \mathrm{M}$ PBS for $24 \mathrm{~h}$. After that the samples were dehydrated in ascending ethanol series, passed through propylene oxide, immersed in propylene oxide and Durcupan, and embedded in Durcupan (Fluka AG, Buchs SG, Switzerland). The resin block was cut into ultrathin sections by a diamond knife in an ultramicrotome. Each section, 50-70 nm thick, was collected on metal mesh 'grids' and stained with electron dense stains before TEM observation.

\section{Statistical analysis}

The distribution of mast cells was quantitated and statistical analysis was performed using Student's $t$-test for parametrical data (SigmaStat ${ }^{\circledR} 11.0$ software package, Systat Software Inc). Differences were considered statistically significant at $\mathrm{P}<0.05$.

\section{RESULTS}

The distribution of observed mast cells in the vicinity of the needle tract in Zusanli $\left(\mathrm{ST}_{36}\right)$ acupoint in two subzones: $\mathrm{I}-$ to 50 $\mu \mathrm{m}$ and II - from 51 to $100 \mu \mathrm{m}$, was uneven (Fig. 1A,B; Fig. 2A-F). Degranulation of some mast cells was observed on section stained by the three methods (TB,

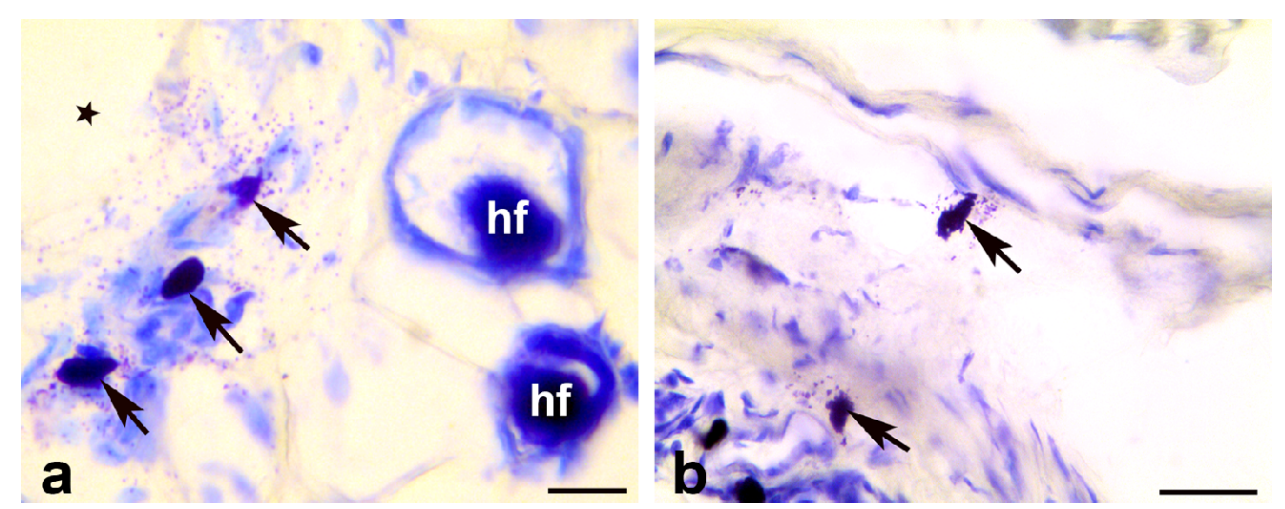

Fig. 1. Toluidine blue-stained sections in the vicinity of the needle tract after acupuncture in acupuncture point $\mathrm{ST}_{36}$ in rats. A. MCs with signs of degranulation (arrow) in the vicinity of needle tract (star) and hair follicles (hf); B. MCs with signs of degranulation (arrow) in the proximity of needle tract after acupuncture. Scale bars $=50 \mu \mathrm{m}$. 


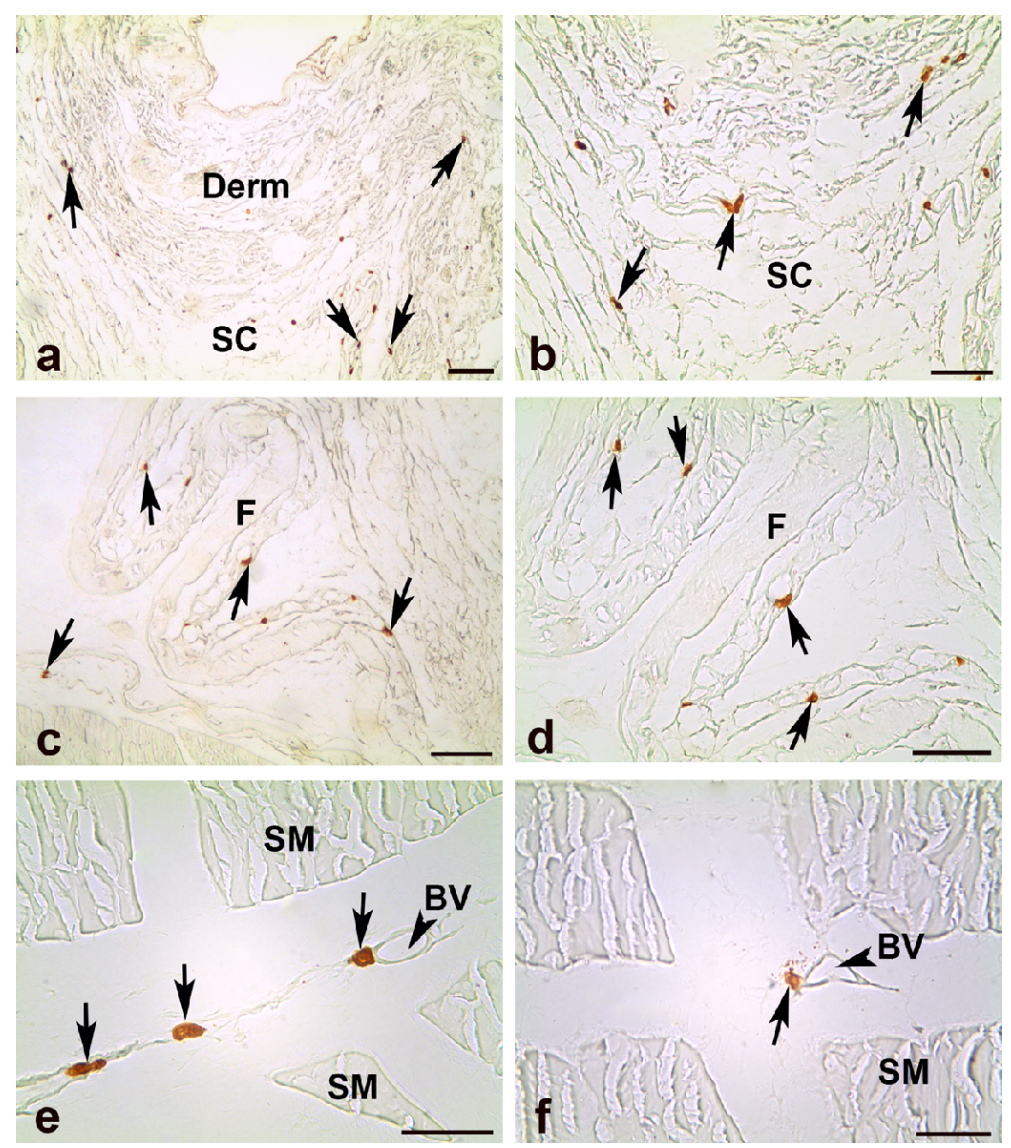

Fig. 2. Bismarck brown-stained MCs in the vicinity of the needle tract after acupuncture in $\mathrm{ST}_{36}$ acupoint in rats. A, B. Distribution of MCs (arrow) in the vicinity of the needle tract after acupuncture in dermis (Derm) and subcutis (SC); C, D. MCs (arrow) in the vicinity of the needle tract after acupuncture next to folded fascia (F); E. MCs (arrow) in the vicinity of needle tract after acupuncture in striated muscle (SM), next to blood vessels (BV); F. Degranulation of MCs (arrow) in the vicinity of the needle tract after acupuncture in striated muscle (SM), next to blood vessels (BV). Scale bars $=50 \mu \mathrm{m}$.

BB and MT). The subcutaneous connective tissue close to the needle tract was thickened and its deeper parts containing mast cells reached the striated muscles (Fig. 3D).

The main MCs aggregations however were found in the border zones: epidermis - dermis, dermis - subcutis, and subcutis - fascia. Obviously, comparatively more MCs were observed in the dermis and subcutis than in the folded fascia and pe- rimysium of striated muscles. Clusters of several MCs were found in close proximity of blood vessels and nerve fibres as well. In zone I, larger number of MCs was established in stratum papillare of the dermis, and on the border dermis - subcutis, where aggregations of blood vessels of different calibres were located (Fig. 2AD). At the dermis level MCs were distributed mainly around blood vessels and close to hair follicles in proximity of the 


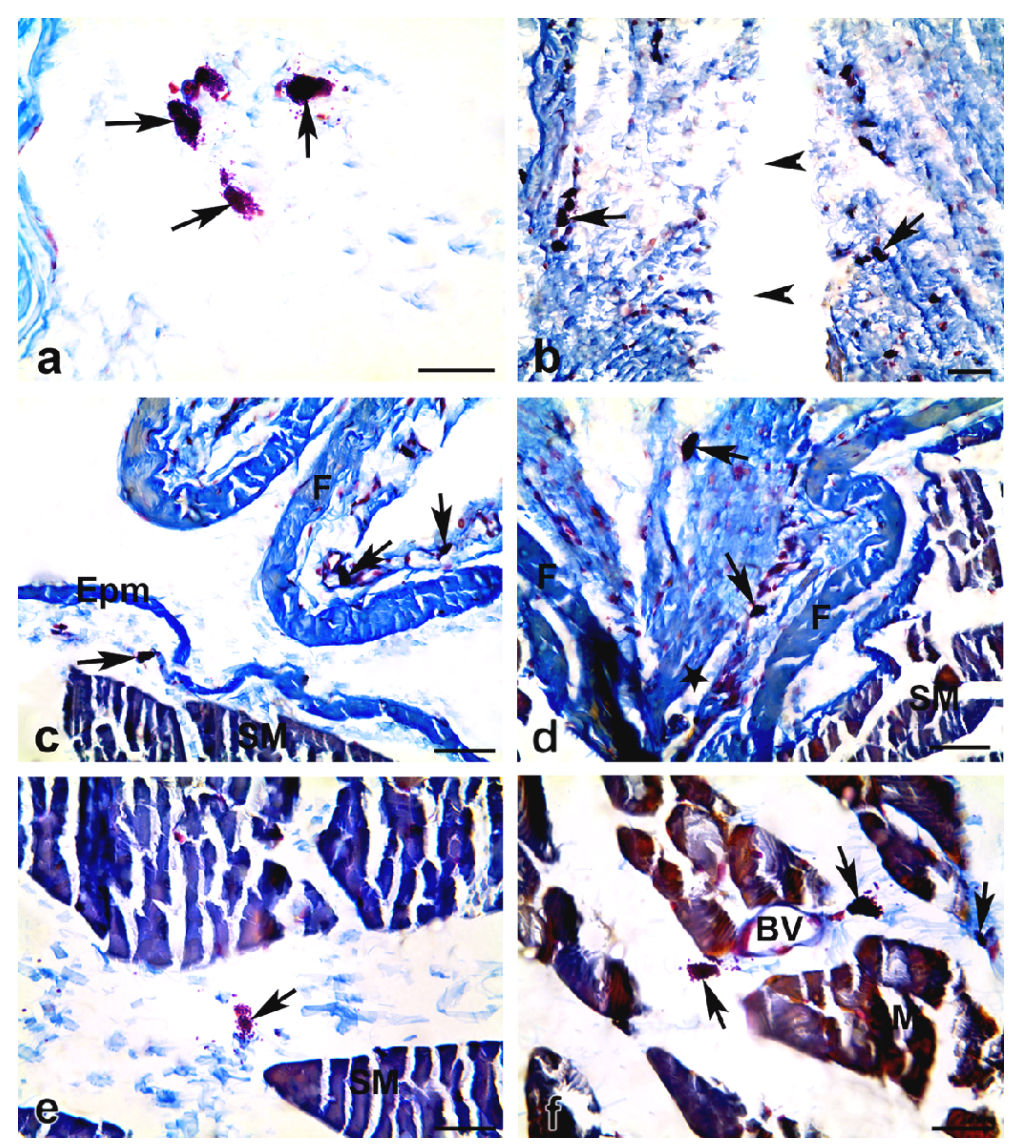

Fig. 3. Mallory's trichrome stained sections of the needle tract vicinity after acupuncture in acupoint $\mathrm{ST}_{36}$ in rats. A. MCs with degranulation (arrow) in the needle tract vicinity after acupuncture. B. MCs with degranulation (arrow) in the needle tract vicinity after acupuncture (arrowhead) in the dermis. C. MCs (arrow) next to folded fascia (F) and epimysium (Epm); D. MCs (arrow) next to folded fascia (F) and needle tract after acupuncture (star); E. MCs with signs of degranulation (arrow) in the vicinity of the needle tract after acupuncture in striated muscle (SM); F. MCs with signs of degranulation (arrow) in the vicinity of needle tract after acupuncture in striated muscle (SM) next to blood vessels (BV). Scale bars $=50 \mu \mathrm{m}$.

needle tract (Fig. 1A; Fig. 4A,B). In the vicinity of the needle tracts within striated muscles MCs were localised into its connective tissue and were less numerous than those found in the dermis and submucosa - they were usually observed as single cells, or in clusters of 3-4, rarely more MCs. Some of MCs showed marked degranulation (Fig. 2F; Fig. 3E,F). De- granulation was also demonstrated by transmission electron microscopy (Fig. 5).

The analysis of the number and distribution of MCs between Zone I and Zone II in "Zusanli" $\left(\mathrm{ST}_{36}\right)$ acupoint showed no statistically significant difference between the two areas ( $>00.05$ ) (Fig. 6). 

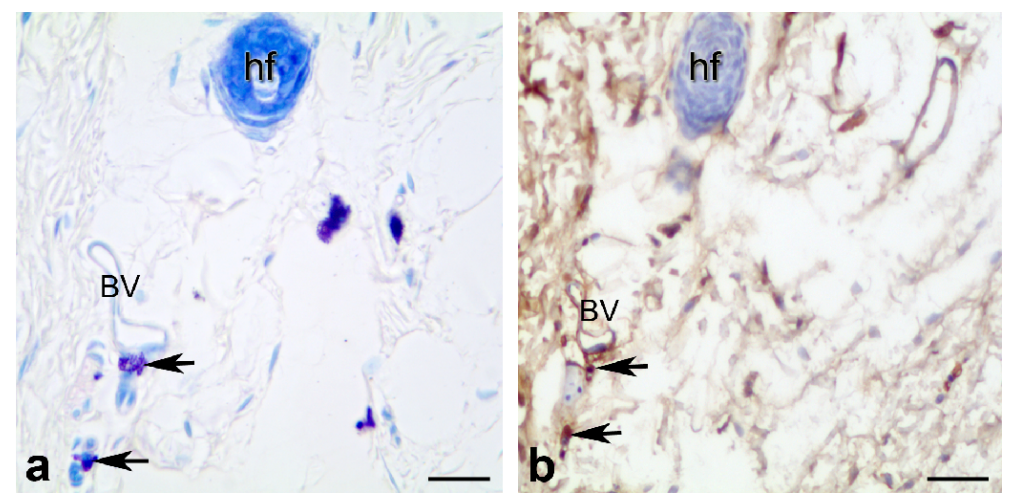

Fig. 4. Mast cells in the vicinity of the needle tract. A. Toluidine blue-stained MCs (arrow) next to blood vessels (BV) and hair follicles (hf). B. Tryptase positive MCs (arrow) next to blood vessels (BV) and hair follicles (hf).

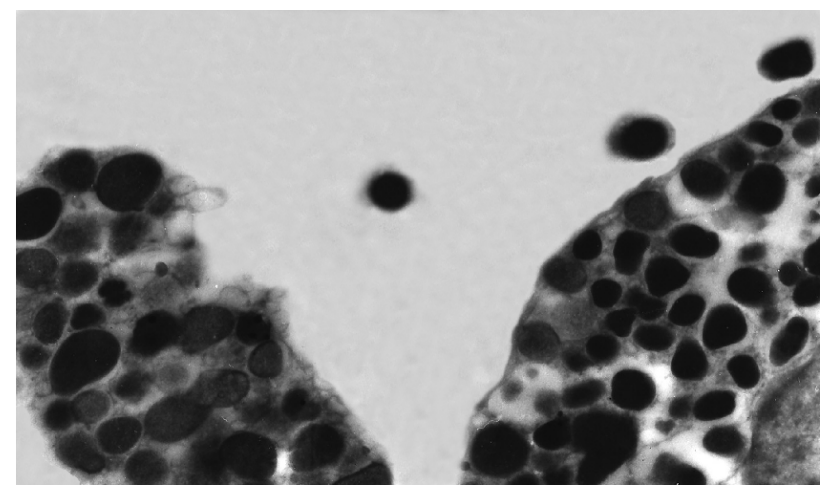

Fig. 5. Electron micrograph showing degranulation of MCs in the needle tract vicinity $(4900 \times)$.

\section{DISCUSSION}

The obtained results convincingly demonstrated MCs presence around the needle tract in $\mathrm{ST}_{36}$ acupoint in rats. However, they were not evenly distributed in the two defined zones - I and II. In contrast to finding of Wu et al. (1980) and Zhang et al. (2005) who reported chemotactic longitudinal migration of MCs along the meridian lines, we did not observe such behaviour. In fact a comparative recruitment of MCs around the needle tract of Zusanli acupoint was also found, which in our opinion was influenced by acupuncture, and especially by retaining needles for a very short time in the tissues of the anaesthesised rats. It could be presumed that a longer stay of the needle into the tissues would most probably increase mast cells number. Obviously, further research is needed to confirm this hypothesis. The degranulation of MCs was clearly demonstrated by all three staining methods. This fact is of special importance for MT staining, which gave very clear and representative results, not only for MCs visualisa- 


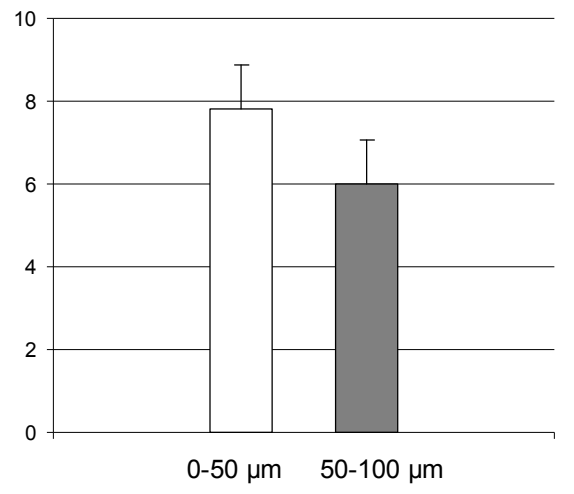

Fig. 6. Mast cells distribution (mean number in an area of $0.25 \mathrm{~mm}^{2} \pm$ SEM) in the tissues around the needle tract after acupuncture in $\mathrm{ST}_{36}$ acupoint in rats: zone I $(0-50 \mathrm{~mm}$ from the needle tract) and zone II (50- $100 \mathrm{~mm}$ from the needle tract).

tion, but for their degranulation, as well. Our data based on the observed degranulation by both light and transmission electron microscopy agree with other findings (Yang \& Waug, 1986; Deng et al., 1996; Luo et al., 2007; Dimitrov, 2012b), but not with the results of Zong et al. (1992) affirming unremarkable influence of acupuncture on the MCs number and degranulation.

Special attention should be paid to MCs participation in tissue repair and healing after acupuncture. Having in mind the opinion of Miller \& Whitting (1964) that MCs located beneath the basal layer of the epidermis in normal rats are involved in wound healing of the skin and our earlier findings (Dimitrov, 2012a,b) about the normal structure of $\mathrm{ST}_{36}$ and it change after acupuncture (thickening and shifting of the connective tissue together with the MCs in it) it could be suggested that MCs take part in tissue repair. Last but not least, our findings may explain the healing effect of acupuncture and the possible involvement of MCs in this effect.

\section{CONCLUSION}

There were no obvious differences in mast cells number and distribution in the vicinity of the needle tract formed by acupuncture in $\mathrm{ST}_{36}$ acupoint in rats. Acupuncture triggered a cascade of reactions ultimately leading to degranulation of closely located mast cells.

\section{REFERENCES}

Cheng, K., X. Shen, G. Ding \& F. Wu, 2009. Relationship between laser acupuncture analgesia and the function of mast cells. Chinese Acupuncture \& Moxibustion, 29, 478-483.

Deng, Y., Z. Fu, H. Dong, Q. Wu \& X. Guan, 1996. Effects of electroacupuncture on the subcutaneous mast cells of zusanli acupoint in rat with unilateral sciatic nerve transection. Acupuncture Research, 21, 46-49.

Dimitrov, N. D., D. P. Sivrev, N.L. Pirovski \& A. Georgieva, 2009. Methods for localization of BAP of the human body. Journal of Biomedical and Clinical Research, 2, 1921.

Dimitrov, N. D., 2012a. Normal morphology of biologically active point $\mathrm{BAP} / \mathrm{ST}_{36}$ rat. Acta Morphologica and Anthropologica, 19, 4-37.

Dimitrov, N. D., 2012b. Morphological changes in biologically active point /BAP/ ST36 after acupuncture in rat. Acta Morphologica and Anthropologica, 19, 30-33.

He, J. \& M. Luo, 2007. Progress in the study on the relationship between effects of acumoxibustion and mast cells in acupoints. Acupuncture Research, 32, 214-216.

He, T. \& Y. Chen, 2010. Advances in studies on the correlation between acupuncturemoxibustion treatment and mast cells. Chinese Acupuncture \& Moxibustion, 30, 84-87.

Li, M., J. Shi, X. Liu, L. Wang, J. Zhang, L. Li $\&$ X. Guan, 2003. Effects of electroacu- 
puncture on the number of subcutaneous mast cells in and beside the acupoint and the inflammatory pain focus in the rat. Chinese Acupuncture \& Moxibustion, 23, 597-601.

Lin, J., H. Huang, G. Ding \& D. Zhang, 2007. Relationship between the function of mast cells and acupuncture analgesia in adjuvant arthritis rats. Acupuncture Research, 32, 16-19.

Luo, M., J. He, Y. Guo \& C. Li, 2007. Effect of electroacupuncture and moxibustion of "Dazhui" (GV14) on the number and distribution of degranulated mast cells in GV14 region. Acupuncture Research, 32, 327-329.

Metcalfe, D. D., D. Baram \& Y. A. Mekori, 1997. Mast cells. Physiological Reviews, 77, 1033-1079.

Miller, L. \& H. W. Whitting, 1964. Mast cells and wound healing of the skin in the rat. Zeitschrift fur Zellforschung und mikroskopische Anatomie, 65, 597-606.

Ming, C., S. Cai, Y. Cai \& T. Ma, 2000. Observation of MC in the deep aponeurosis under the fluorescent microscope by electric needling in "Zusanli". Acupuncture Research, 25, 51-53.

White, A., M. Cummings \& J. Fishie, 2008. How to locate acupuncture points. In: $A n$ Introduction to Western Medical Acupuncture, ed. K. Morley, Churchill Livingstone Elsevier, Edinburgh. pp. 185-189.

Wu, J. L., D. H. Chai, D. Cai \& A. Zong, 1980. Observation on mast cells in subcutaneous connective tissue at points in albino rats. Acta Anatomica Sinica, 11, 308-312.
Yang Y. \& P. Waug, 1986. Morphological observation on the effect of acupuncture on mast cells at the zusanli point. $\mathrm{Acu}$ puncture Research, 11, 298-302.

Zhang D., G. Ding, X. Shen, W. Yao \& J. Lin, 2005. Study on the correlation between meridian acupoints and mast cells. Acupuncture Research, 3, 115-119.

Zhang D., G. H. Ding, X. Y. Shen, W. Yao, Z. Y. Zhang, Y. Q. Zhang, J. Y. Lin \& Q. Gu, 2008. Role of mast cells in acupuncture effect: A pilot study. Explore (NY), 4, 170 177.

Zong A., X. Shi \& F. Zhang, 1992. Effects of electro-acupuncture on fascial mast cells in "Zusanli" acupoint area of rabbits. Journal of Zhengzhou University (Science Medical), 27, 226-229.

Paper received 01.06.2017; accepted for publication 05.06.2017

\section{Correspondence:}

Nikolay Dimitrov MD, PhD

Department of Anatomy

Faculty of Medicine

Trakia University 11, Armejska Str. 6003 Stara Zagora, Bulgaria e-mail: nikolaydd@abv.bg 